Abanico Veterinario. Enero-Diciembre 2020; 10(1):1-9. http://dx.doi.org/10.21929/abavet2020.4 Artículo Original. Recibido: 02/10/2019. Aceptado: 22/01/2020. Publicado: 15/02/2020.

\title{
Efecto de los antioxidantes Trolox y Crocina sobre la criopreservación del semen de ovino Pelibuey
}

Antioxidant effect of Trolox and Crocin on the cryopreservation of Pelibuey rams semen

$$
\begin{aligned}
& \text { Rodríguez-Gutiérrez Itzel }{ }^{2} \text {, Domínguez-Rebolledo Álvaro }{ }^{1^{*}}(\mathbb{D} \text {, Loeza-Concha } \\
& \text { Henry }^{3} \text {, Castellanos-Zacarías Carlos }{ }^{2} \text {, Tun-Moo Maximiliano }{ }^{2} \text { iD, Ramón- } \\
& \text { Ugalde Julio }{ }^{2}
\end{aligned}
$$

${ }^{1}$ Instituto Nacional de Investigaciones Forestales, Agrícolas y Pecuarias, Campo Experimental Mocochá. Km. 25 Antigua carretera Mérida-Motul. C.P. 97454. Mocochá, Yucatán. México. ${ }^{2}$ Tecnológico Nacional de México. Instituto Tecnológico de Conkal. Antigua Carretera Mérida-Motul. km 16.3, C.P. 97345, Conkal, Yucatán. México. ${ }^{3}$ Colegio de Postgraduados, Campus Campeche. Carretera Haltunchén-Edzná. km 17.5, C.P. 24450; Sihochac, Champotón, Campeche. México. *Autor para correspondencia: Álvaro Domínguez Rebolledo. Itzel.rgutierrez@outlook.com, dominguez.alvaro@inifap.gob.mx, henryloeza_21@yahoo.com, carlos.castellanos@itconkal.edu.mx, maxtun_14@hotmail.com,julio.ramon9@gmail.com.

\section{RESUMEN}

El objetivo del estudio fue evaluar el efecto de los antioxidantes Trolox y Crocina en el diluyente de congelación sobre la criopreservación del semen de ovino Pelibuey. Los eyaculados obtenidos se mezclaron, dividieron y congelaron en 3 tratamientos: T1: Trolox 1mM; T2: Crocina 1mM; TT: Testigo (sin antioxidante). Se analizó a las 0 horas (descongelación) y 6 horas a $37^{\circ} \mathrm{C}$, la motilidad total (MT) y progresiva (MP), la viabilidad espermática, la actividad mitocondrial, la integridad de los acrosomas y de la membrana de la cola (Host). Los datos se analizaron con un ANOVA para un diseño completamente al azar y una prueba de Tukey para la comparación de medias. En ninguna de las dos horas se encontraron diferencias significativas en los tratamientos $(P>0.05)$. Sin embargo, de 0 a $6 \mathrm{~h}$, la MT se comportó de forma similar en el T1 y T2, pero no así en el TT $(\mathrm{P}<0.01)$, por el contrario, la integridad de los acrosomas en los tres tratamientos fue similar $(P>0.05)$. La MP, viabilidad, actividad mitocondrial y Host disminuyeron con el tiempo de forma similar en los tres tratamientos. Los antioxidantes Trolox y Crocina a $1 \mathrm{mM}$ en el diluyente de congelación, permiten mantener la MT y la integridad de los acrosomas durante 6 h de incubación a 37 ${ }^{\circ} \mathrm{C}$.

Palabras clave: Radicales libres, diluyente, esperma y congelación.

\section{ABSTRACT}

The aim of this study was to assess the effect of two antioxidants (Trolox and Crocin) added to the freezing extender on the cryopreservation of Pelibuey ram semen. Semen samples were pooled, split and frozen into 3 treatments: T1: Trolox 1mM; T2: Crocin 1mM; TT: Control (without antioxidant). Total motility (MT), progressive motility (PM), sperm viability, mitochondrial activity, acrosomes integrity, and plasma membrane integrity of the tail (Host) were assessed at $0 \mathrm{~h}$ (thawed), and at 6 hours at $37^{\circ} \mathrm{C}$. The results were analyzed with ANOVA, followed by Tukey's comparison test. At $0 \mathrm{~h}$ and $6 \mathrm{~h}$, no differences were found $(P>0.05)$ in all the parameters evaluated between treatments, however, from 0 to 6 hours, the MT was similar in the T1 and T2, but not in the TT $(P<0.01)$, on the contrary, acrosome integrity in the three treatments was similar $(P>0.05)$. MP, viability, mitochondrial activity, and Host dropped through time in a similar way in the three treatments. The addition of the antioxidants Trolox and Crocin at $1 \mathrm{mM}$ to the freezing extender allows maintaining the total motility and acrosome integrity during $6 \mathrm{~h}$ of incubation at 37 ${ }^{\circ} \mathrm{C}$.

Keywords: Free radicals, extender, sperm, freeze. 


\section{INTRODUCCIÓN}

La criopreservación de los espermatozoides es una herramienta importante para conservar el material genético y mantener la diversidad genética en especies silvestres y domésticas (Lermen et al., 2009). Sin embargo, se sabe que los procesos de congelación-descongelación de semen empleados en la mayoría de las especies de mamífero ocasionan la muerte de un gran número de células (Watson, 2000).

En el ovino, a pesar de que un porcentaje relativamente alto (40-60 \%) de espermatozoides conservan su motilidad después de la descongelación, sólo alrededor del 20-30 \% permanecen biológicamente intactos (Salamon y Maxwell, 2000). Esto es debido que durante el proceso de refrigeración y criopreservación se presenta un incremento en los niveles de las especies reactivas de oxígeno (ROS), los cuales producen cambios biofísicos y bioquímicos en la membrana de los espermatozoides (Chatterjee et al., 2001; Kankofer et al., 2005) e incluso daño en su ADN (Aitken, 1999; Agarwal et al., 2003), afectando la viabilidad y su capacidad fecundante. En los últimos años los protocolos de criopreservación han sido mejorados sustancialmente con la adición de compuestos con propiedades antioxidantes, repercutiendo positivamente en la mejora de la calidad del semen descongelado (Peña et al., 2005; Thuwanut et al., 2008; Domínguez-Rebolledo et al., 2010; Mata-Campuzano et al., 2015).

Los antioxidantes juegan un papel importante en la protección de las células espermáticas, frente a los efectos dañinos que producen las ROS (Halliwell, 1997). La función de un antioxidante se basa en donar electrones a otras moléculas que tiene uno o más electrones perdidos (ROS), y así evitar que se produzcan alteraciones en las moléculas de los lípidos, proteínas y del ADN espermático (Tremellen et al., 2008).

El antioxidante Trolox es un análogo de la vitamina E, soluble en agua, y se ha utilizado en una gran variedad de sistemas celulares para evitar los efectos de las ROS (Halliwel, 1994; Michielset al., 1994). Se ha observado que la adición de este antioxidante en el medio de congelación, mejora la calidad de los espermatozoides descongelados de cerdo (Peña et al., 2005); así como la viabilidad espermática de semen descongelado en ovinos de la raza Churra (Mata-Campuzano et al., 2015). En muestras espermáticas procedentes del epidídimo (postmortem) de ciervo lbérico, se demostró que este antioxidante es capaz de disminuir las cantidades de ROS y de la lipoperoxidación; además de proteger los acrosomas y el ADN espermático (Domínguez-Rebolledo et al., 2010). Thuwanut et al. (2008) observaron que la adición del Trolox al diluyente de congelación, mejoraba la motilidad y la viabilidad en los espermatozoides de epidídimo de gato en la descongelación.

El antioxidante Crocina (glucosil éster de crocetina), es un carotenoide poco soluble en agua, que se encuentra en el pigmento amarillo del azafrán (Crocussativus). Se ha 
demostrado que la adición de este antioxidante en el medio de incubación de semen, mejora la calidad de los espermatozoides descongelados del ciervo lbérico (DomínguezRebolledo et al., 2010) y toro (Sapanidou et al., 2014). En semen de gallos, mejora la viabilidad, motilidad, actividad mitocondrial y reduce la lipoperoxicación a la descongelación (Mehdipour et al., 2019).

Por lo anterior, el objetivo del presente estudio fue evaluar el efecto de la adición de los antioxidantes Trolox y Crocina al medio de congelación, sobre las características espermáticas post-criopreservación en ovinos de la raza Pelibuey.

\section{Ubicación}

\section{MATERIAL Y MÉTODOS}

El estudio se realizó en el Banco de Germoplasma Ovino Pelibuey y Blackbelly, del Instituto Nacional de Investigaciones Forestales, Agrícolas y Pecuarias (INIFAP), Campo Experimental Mocochá.

\section{Animales}

Se utilizaron 4 ovinos adultos (2.5 años), de la raza Pelibuey, de 40 a $45 \mathrm{~kg}$ de peso vivo, con una condición corporal de 3 a 3.5; a los cuales previamente se evaluó su estado de salud y calidad espermática.

\section{Obtención de muestras espermáticas}

Se obtuvieron 36 eyaculados de los 4 sementales, mediante una vagina artificial, y con la ayuda de una oveja que sirvió como maniquí.

\section{Dilución espermática}

Los eyaculados obtenidos fueron mezclados (pool) y diluidos en Triladyl $\mathbb{B}+20 \%$ yema de huevo, a una concentración final de $400 \times 10^{6}$ espermatozoides $/ \mathrm{ml}$. Posteriormente, las muestras fueron divididas en 3 tratamientos: T1: Trolox 1mM; T2: Crocina 1mM; TT: Testigo (sin antioxidante), para luego ser envasadas en pajillas de $0.25 \mathrm{ml}$.

\section{Congelación de semen}

La congelación de las muestras se realizó colocando las pajillas a $4 \mathrm{~cm}$ sobre la superficie del nitrógeno líquido (LN2), durante 10 minutos. Inmediatamente después las pajillas se sumergieron en LN2 y se almacenaron hasta su evaluación.

\section{Descongelación de semen}

El procedimiento de descongelación se realizó mediante la inmersión de las pajillas en un baño maría a $37^{\circ} \mathrm{C}$ durante 30 segundos; posteriormente las muestras fueron evaluadas a la $0 \mathrm{~h}$ (descongelación), a las 6 horas de incubación a $37^{\circ} \mathrm{C}$. 


\section{Concentración espermática}

Se diluyó una pequeña fracción de la muestra de semen $(5 \mu \mathrm{L})$, en $995 \mu \mathrm{L}$ agua destilada; después se tomó $9 \mu \mathrm{L}$ de la muestra diluida en agua y se colocó en cada uno de los dos lados de la cámara bücker, para estimar su concentración, con el módulo de concentración del sistema CASA (ISAS®v1 (Proiser R + D, Valencia, España). Se capturaron 4 campos de cada lado de la cámara y se obtuvo la concentración espermática.

\section{Motilidad espermática}

La motilidad se analizó con el sistema CASA, colocando $5 \mu \mathrm{L}$ de semen descongelado y diluido a $\sim 30 \times 10^{6} / \mathrm{ml}$ de espermatozoides, sobre una cámara de recuento Makler ${ }^{\circledR}$ (Sefi Medical Instruments, Haifa, Israel), precalentada a $37^{\circ} \mathrm{C}$; y se procedió a la captura de al menos cinco campos con un mínimo de 300 espermatozoides/muestra. Los parámetros de motilidad evaluados fueron: Motilidad Total (MT\%) y Motilidad Progresiva (MP\%).

\section{Viabilidad espermática}

Se evaluó mediante la tinción de SYBR14-IP (Live/Dead® kit L-7011, Invitrogen ${ }^{T M}$ ), añadiendo $1 \mu \mathrm{L}$ de SYBR14 $(10 \mu \mathrm{M})$ y de IP $(12 \mu \mathrm{M})$ de la solución stock en $100 \mu \mathrm{L}$ de muestra espermática, diluida en solución salina (PBS); y se dejó incubar durante 10 minutos a $37^{\circ} \mathrm{C}$. Posteriormente se colocaron $5 \mu \mathrm{L}$ de la muestra entre un portaobjeto y cubreobjeto precalentado a $37^{\circ} \mathrm{C}$, y se procedió a su evaluación por medio de un microscopio de epifluorescencia (LWScientific i40-ADN); contabilizando 200 espermatozoides, los cuales presentaban fluorescencia roja (muertos), y de color verde los que estaban vivos.

\section{Integridad del acrosoma}

Se evaluó mediante la tinción de FITC-PSA $\left(100 \mu \mathrm{g} / \mathrm{ml}\right.$, L-0770, Sigma-Aldrich $\left.{ }^{\mathrm{TM}}\right)$, añadiendo $5 \mu \mathrm{L}$ de la solución stock en $100 \mu \mathrm{L}$ de muestra espermática diluida en PBS y se dejó incubar en la oscuridad durante 30 minutos a $37^{\circ} \mathrm{C}$. Inmediatamente después se colocaron $5 \mu \mathrm{L}$ de la muestra entre un portaobjeto y cubreobjeto. Finalmente se contabilizaron 200 espermatozoides con un microscopio de epifluorescencia, los cuales presentaban fluorescencia de color verde (acrosomas dañados), y sin fluorescencia de los acrosomas intactos.

\section{Actividad mitocondrial}

Se analizó con la tinción JC-1 (153 $\mu \mathrm{M}$, Molecular Probes ${ }^{\circledR} \mathrm{T}-3168$, Invitrogen ${ }^{\mathrm{TM}}$ ), añadiendo $1 \mu \mathrm{L}$ de la solución stock en $100 \mu \mathrm{L}$ de muestra espermática diluida en PBS y se dejó incubar en la oscuridad durante 10 minutos a $37^{\circ} \mathrm{C}$. Después, se colocaron $5 \mu \mathrm{L}$ de la muestra sobre un portaobjeto y cubreobjeto, y se contabilizaron 200 espermatozoides mediante un microscopio de epifluorescencia, los cuales presentaban 
en la parte intermedia del flagelo una fluorescencia de color naranja (mitocondrias activas), y de color verde las mitocondrias inactivas.

\section{Integridad de la membrana plasmática de la cola (HOST)}

Se realizó diluyendo $5 \mu \mathrm{L}$ la muestra espermática en $50 \mu \mathrm{L}$ de solución de endósmosis ( $0.735 \mathrm{~g}$ de citrato de sodio dihidratado y $1.351 \mathrm{~g}$ de fructosa en $100 \mathrm{ml}$ de agua destilada) a $100 \mathrm{mOsm} / \mathrm{L}$ y se dejó incubar durante $37^{\circ} \mathrm{C}$. Posteriormente se colocaron $5 \mu \mathrm{L}$ de la muestra sobre un portaobjeto y cubreobjeto y se contabilizaron 200 espermatozoides con un microscopio de contraste de fases, los cuales presentaban colas enrolladas (endósmosis positiva), y no enrolladas (endósmosis negativa).

\section{Análisis estadístico}

Las variables expresadas como porcentajes (motilidad total, motilidad progresiva, viabilidad, actividad mitocondrial, acrosomas intactos y Host), fueron transformadas al arcoceno $\sqrt{ }($ variable)/100 antes de su análisis. Posteriormente se analizó con un modelo lineal general (GLM) con procedimiento PROC GLM; y para encontrar las diferencias estadísticas entre tratamientos se utilizó la prueba de Tukey a $P \leq 0.05$, a través del paquete estadístico del Statistical Analysis System (SAS Inst. Inc., 2003).

\section{RESULTADOS Y DISCUSIÓN}

A las 0 y $6 \mathrm{~h}$ de incubación, ninguno de los parámetros evaluados presentó diferencias $(P>0.05)$ entre los tratamientos (cuadro 1). Estos resultados difieren a los obtenidos por Thuwanut et al. (2008) con espermatozoides epididimarios de gato suplementados con el antioxidante Trolox a 1mM; ya que la MT, MP y la viabilidad espermática mejoraron a la descongelación. Si bien son especies distintas, la diferencia en resultados podría atribuirse a que los espermatozoides provenientes del epidídimo de gato por castración, nunca han estado en contacto con las secreciones de las glándulas que forman el plasma seminal, que es donde se encuentra la mayor defensa antioxidante (Chen et al., 2003); por lo que existen diferencias en la congelación entre espermatozoides procedentes de eyaculados y epididimarios (Gilmore et al., 1998), que afectan la sensibilidad al enfriamiento, como la resistencia a la congelación (Schmehl et al., 1986). En este sentido, el Trolox pudo haber actuado directamente y sin competencia alguna sobre los espermatozoides del epidídimo, similar a lo observado por Domínguez-Rebolledo et al. (2007), con plasma seminal ovino sobre el semen descongelado.

Por otra parte, en sinergia con los antioxidantes presentes en el plasma seminal, las muestras seminales utilizadas en este estudio, pudieron verse alteradas, ocasionando que su efecto disminuya. Asimismo, se ha reportado que la suplementación del diluyente con Trolox a 1mM, dependiendo del tipo de aditivo utilizado en el diluyente, puede mejorar o no la viabilidad del semen congelado de ovinos de la raza Churra (Mata- 
Campuzano et al., 2015); es decir, cuando el diluyente era elaborado a base de lecitina de soya, el antioxidante Trolox presentaba una mayor viabilidad en las muestras descongeladas; mientras que cuando se utilizaba un diluyente a base de tris, como el que se utilizó en este estudio (Triladyl®), la viabilidad en los espermatozoides no mejoraba. La soya contiene isoflavonas, que actúan como antioxidantes; los cuales pudieron haber ejercido algún tipo de protección sobre los espermatozoides durante el proceso de la congelación-descongelación o en combinación con el Trolox, produciendo una sinergia que coadyuvo a potenciar los efectos benéficos en los espermatozoides. Con la adición del antioxidante Crocina, se ha reportado una mayor viabilidad, motilidad, actividad mitocondrial y reducción de la lipoperoxidación, en muestras espermáticas de gallo a la descongelación (Mehdipour et al., 2019).

Tabla 1. Porcentajes (\%) de los parámetros espermáticos evaluados a la $0 \mathrm{~h}$ y a la 6 (media \pm error estándar).

\begin{tabular}{|c|c|c|c|c|c|c|c|}
\hline Hora & Tratamiento & $\begin{array}{c}\text { Motilidad } \\
\text { Total }\end{array}$ & $\begin{array}{l}\text { Motilidad } \\
\text { Progresiva }\end{array}$ & Viabilidad & $\begin{array}{c}\text { Actividad } \\
\text { mitocondrial }\end{array}$ & $\begin{array}{c}\text { Acrosomas } \\
\text { intactos }\end{array}$ & Host \\
\hline \multirow{4}{*}{0} & T1 & $57.5 \pm 5.3^{\mathrm{aA}}$ & $17.1 \pm 1.6^{\mathrm{aA}}$ & $39.5 \pm 3.9 \mathrm{aA}$ & $50.7 \pm 6.2^{\mathrm{aA}}$ & $49.6 \pm 6.4^{\mathrm{aA}}$ & $23.6 \pm 3.1 \mathrm{aA}$ \\
\hline & $\mathrm{T} 2$ & $49.9 \pm 5.6^{\mathrm{aA}}$ & $15.8 \pm 2.2 \mathrm{aA}$ & $45.2 \pm 5.2^{\mathrm{aA}}$ & $42.3 \pm 5.4^{\mathrm{aA}}$ & $44.6 \pm 6.1 \mathrm{aA}$ & $23.4 \pm 3.1 \mathrm{aA}$ \\
\hline & TT & $58.6 \pm 3.9^{a A}$ & $15.3 \pm 2.2^{a A}$ & $41.4 \pm 5.2^{\mathrm{aA}}$ & $40.5 \pm 6.0^{a A}$ & $47.7 \pm 6.1 \mathrm{aA}$ & $19.3 \pm 2.1 \mathrm{aA}$ \\
\hline & $\mathrm{T} 1$ & $36.6 \pm 7.0^{\mathrm{aA}}$ & $8.6 \pm 7.0^{\mathrm{aB}}$ & $24.6 \pm 4.0^{a B}$ & $15.7 \pm 1.9 \mathrm{aB}$ & 46. $0 \pm 2.7 \mathrm{aA}$ & $10.2 \pm 1.0 \mathrm{aB}$ \\
\hline & $\mathrm{T} 2$ & $39.2 \pm 5.8^{\mathrm{aA}}$ & $8.9 \pm 5.8 \mathrm{aB}$ & $22.8 \pm 2.7 \mathrm{aB}$ & $17.1 \pm 1.5^{\mathrm{aB}}$ & $43.7 \pm 4.0^{\mathrm{aA}}$ & $13.1 \pm 1.1 \mathrm{aB}$ \\
\hline & TT & $26.7 \pm 4.3^{\mathrm{aB}}$ ** & $8.2 \pm 4.3 \mathrm{aB}$ & $27.2 \pm 4.0 \mathrm{aB}$ & $20.0 \pm 2.2^{a b}$ & $41.7 \pm 3.5^{\mathrm{aA}}$ & $12.4 \pm 1.2^{\mathrm{aB}}$ \\
\hline
\end{tabular}

T1: Trolox 1mM; T2: Crocina 1mM; TT: Testigo (sin antioxidante). (ab) Literales diferentes dentro de la misma columna indican diferencias significativas entre tratamientos dentro de cada hora $(P<0.05)$. $(A B)$ Literales diferentes dentro de la misma columna indican diferencias significativas $\left(P<0.01^{* *}\right)$ entre el mismo tratamiento a través del tiempo.

A través del tiempo de incubación ( 0 a 6 horas), la MT se comportó de forma similar en el T1 y T2, pero no así en el TT $(\mathrm{P}<0.01)$; por el contrario, la integridad de los acrosomas en los tres tratamientos resultó similar $(P>0.05)$. La MP, viabilidad, actividad mitocondrial y Host disminuyeron a través del tiempo de forma similar en los tres tratamientos (tabla 1). Los resultados son similares a los reportados por Domínguez-Rebolledo et al. (2010) en muestras espermáticas de epidídimo descongeladas del ciervo Ibérico, donde la MT y la integridad de los acrosomas se mantenían durante la incubación a $37^{\circ} \mathrm{C}$ con el antioxidante Trolox y Crocina a un 1mM. Asimismo, Sapanidou et al. (2014) observó que la motilidad, viabilidad y la integridad de los acrosomas en espermatozoides de bovinos se mantenía durante la incubación a $37^{\circ} \mathrm{C}$ con $1 \mathrm{mM}$ de Crocina. A la vista, de los resultados tras $6 \mathrm{~h}$ de incubación donde el T1 y T2 mantuvieron la MT y la integridad de los acrosomas, se puede decir que los antioxidantes probados son eficientes y recomendables para su incorporación al diluyente previo a la congelación; de tal modo, que la dosis seminal estará vigente en el tracto genital, tras la inseminación hasta el momento cercano a la ovulación. 


\section{CONCLUSIÓN}

La adición de los antioxidantes Trolox y Crocina a $1 \mathrm{mM}$ en el diluyente de congelación, permiten mantener la motilidad total y la integridad de los acrosomas durante $6 \mathrm{~h}$ de incubación a $37^{\circ} \mathrm{C}$.

\section{AGRADECIMIENTO}

Al Consejo Nacional de Ciencia y Tecnología (CONACYT), por el financiamiento otorgado esta investigación por medio del proyecto de Ciencia Básica 164592 y por el proyecto Fiscal No. 1513272874 del INIFAP.

\section{LITERATURA CITADA}

AGARWAL A, Saleh RA, Bedaiwy MA. 2003. Role of reactive oxygen species in the pathophysiology of human reproduction. Fertility and Sterility. 79(4): 829-843. ISSN: 0015-0282. http:/dx.doi.org/10.1016/S0015-0282(02)04948-8

Aitken RJ. 1999. The Amoroso Lecture. The human spermatozoon-a cell in crisis?. Journal of Reproduction and Fertility. 115(1): 1-7. ISSN: 2228-5482. http:/dx.doi.org/10.1530/jrf.0.1150001

CHATTERJEE S, Gagnon C. 2001. Production of reactive oxygen species by spermatozoa undergoing cooling, freezing, and thawing. Molecular Reproduction and Development. 59(4): 451-458. ISSN: 1098-2795. http:/dx.doi.org/10.1002/mrd.1052

CHEN H, Chow, PH, Cheng SK, Cheung AL, Cheng LY, WS O. 2003. Male genital tract antioxidant enzymes: Their source, function in the female, and ability to preserve sperm DNA integrity in the golden hamster. Journal of Andrology. 24(5):704-711. ISSN:19394640. http:/dx.doi.org/10.1002/j.1939-4640.2003.tb02730.x

DOMíNGUEZ-REBOLLEDO AE, Fernando-Santos MR, Bisbal A, Ros-Santaella JL, Ramon M, Carmona M, Martínez-Pastor F, Garde JJ. 2010. Improving the effect of incubation and oxidative stress on thawed spermatozoa from red deer by using different antioxidant treatments. Reproduction and Fertility and Development. 22(5): 856-870. ISSN: 1031-3613. http:/dx.doi.org/10.1071/RD09197

DOMínGUEZ-REBOLLEDO A, Sierra NL, Tamayo C A, Loria AA, Denis ES, Oses BR, Parra GE, Monsreal PL, Ugalde RJ. 2007. Fertility in hair sheep inseminated with freeze spermatozoa rediluted with seminal plasma. Revista Científica. 2007;17:73-76. http://produccioncientificaluz.org/index.php/cientifica/article/view/15259/15234 
GILMORE J, MC Gann I, Ashwoth E, Acker J, Raath J, Bush M, Critser J. 1998. fundamental cryobiology of selected african mammalian spermatozoa and its role in biodiversity preservation through the development of genome resource banking. Animal Reproduction Science. 53(1-4): 277-297. ISSN: 0378-4320. http:/dx.doi.org/10.1016/S0378-4320(98)00118-3

HALLIWEL B. 1994. Free radicals and antioxidants: a personal view. Nutrition Reviews. 52(8): 253-265. ISSN: 0029-6643. http:/dx.doi.org/10.1111/j.1753-4887.1994.tb01453.x

HALLIWELL B. 1997. Antioxidants: The basics-what they are and how to evaluate them. Advances in Pharmacology. 38: 3-20. ISSN: 1054-3589. http:/dx.doi.org/10.1016/S10543589(08)60976-X

KANKOFER M, Kolm G, Aurich J, Aurich C. 2005. Activity of glutathione peroxidase, superoxide dismutase and catalase and lipid peroxidation intensity in stallion semen during storage at $5 \stackrel{\circ}{\circ}$. Theriogenology. 63(5): 1354-1365. ISSN: 0093-691X: http:/dx.doi.org/10.1016/j.theriogenology.2004.07.005

LERMEN D, Blömeke B, Browne R, Clarke A, Dyce PW, Fixemer T, Fuhr GR, Holt WV, Jewgenow K, Lloyd RE, Lotters S, PaulusM, Reid GM, Rapoport DH, Rawson D, Ringleb J, Ryder OA, Sporl G, Schmitt T, Veith M, Muller P. 2009. Cryobanking of viable biomaterials: Implementation of new strategies for conservation purposes. Molecular Ecology. 18(6): 1030-1033. ISSN: 1365-294X. http:/dx.doi.org/10.1111/j.1365294X.2008.04062.x

MATA-CAMPUZANO M, Álvarez-Rodríguez M, Álvarez M, Tamayo-Canul J, Anel L, Paz P, Martínez-Pastor F. 2015. Post-Thawing Quality and Incubation Resilience of Cryopreserved Ram Spermatozoa are Affected by Antioxidant Supplementation and Choice of Extender. Theriogenology. 83(4): 520-528. ISSN: 0093-691X. http:/dx.doi.org/10.1016/j.theriogenology.2014.10.018

MEHDIPOUR M, Kia HD, Najafi A. 2019. Effect of Crocin and Naringenin Supplementation in Cryopreservation Medium on Post-Thawed Rooster Sperm Quality and Expression of Apoptosis Associated Genes. Biorxiv. 1-26. https://doi.org/10.1101/846758

MICHIELS C, Raes M, Toussaint O, Remacle J. 1994. Importance of Se-glutathione peroxidase, catalase, and Cu/Zn-SOD for cell survival against oxidative stress. Free Radicals Biology. 17(3): 235-248. ISSN: 0891-5849. http:/dx.doi.org/10.1016/08915849(94)90079-5

PEÑA FJ, Johannison A, Wallgren M, Rodríguez-Martinez H. 2005. A new and simple method to evaluate early membrane changes in frozen- thawed boar spermatozoa. 
International journal of Andrology. 28(2): 107-114.ISSN:1939-4640: http:/dx.doi.org/10.1111/j.1365-2605.2005.00512.x

SALAMON S, Maxwell WM. 2000. Storage of ram semen. Animal Reproduction Science. 62(1-3): 77-111. ISSN: 0378-4320. http:/dx.doi.org/10.1016/s0378-4320(00)00155-x

SAPANIDOU VG, Margaritis L, Siahos N, Arsenopoulos K, Dragatidou E, Taitzoglou LA, Zervos LA, Theodoridis A, Tsantarliotou MP. 2014. Antioxidant effect of a polyphenol-rich grape pomace extract on motility, viability and lipid peroxidation of thawed bovine spermatozoa. Journal of Biological Research. 21(19): 1-6. ISSN: 2284-0230. http:/dx.doi.org/10.1186/2241-5793-21-19

SCHMEHL MK, Vazquez I, Graham EF. 1986. the effects of nonpenetrating cryoprotectans added to test-yolk-glycerol extender on the post-thawing motility of ram

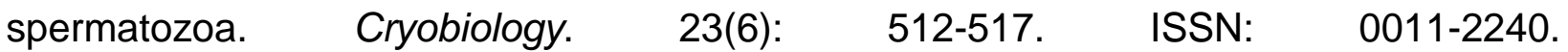
http:/dx.doi.org/10.1016/0011-2240(86)90060-X

S.A.S. Institute Inc. 2003. SAS/STAT user's Guide. Version 6. Fourth Edition. Vol. 1. Carry, NC.

THUWANUT P, Chatdarong K, Techakumphu, M, Axner E. 2008. The effect of antioxidants on motility, viability, acrosome integrity and DNA integrity of frozen-thawed epididymal cat spermatozoa. Theriogenology. 70(2): 233-240. ISSN: 0093-691X. http:/dx.doi.org/10.1016/j.theriogenology.2008.04.005

TREMELLEN K. 2008. Oxidative stress and male infertility: a clinical perspective. Human Reproduction. 14(3): 243-258. ISSN: http:/dx.doi.org/10.1093/humupd/dmn004

WATSON PF. 2000. The causes of reduced fertility with cryopreserved semen. Animal reproduction Science. 60-61: 481-492. ISSN: 0378-4320. http:/dx.doi.org/10.1016/S0378-4320(00)00099-3 